\title{
Propuesta para el manejo ecoeficiente de leña en la agroindustria del cardamomo y su contribución al desarrollo rural
}

\author{
Juan Ramón Ponce Kress \\ Maestría en Desarrollo Rural \\ Centro Universitario del Norte \\ Universidad de San Carlos de Guatemala \\ Gustavo Adolfo García Macz \\ Licenciado en Ciencias Agrícolas \\ M. Sc. en Gerencia para el Desarrollo Sostenible \\ ingusma@gmail.com \\ Profesor Titular \\ Centro Universitario del Norte \\ Universidad de San Carlos de Guatemala
}

Fecha de recepción: 05/05/2017 Fecha de aceptación: 18/07/2017

\begin{abstract}
Resumen
El estudio se realizó en los municipios de Cobán y San Pedo Carchá, ambos del departamento de Alta Verapaz, con la finalidad de analizar y caracterizar al sector agroindustrial que utiliza leña para el secado del cardamomo (Elettaria cardamomum). El cuestionario fue el instrumento utilizado para obtener datos válidos y confiables, necesarios para alcanzar los objetivos planteados. En dicho estudio se identificó como informantes clave a los propietarios de las agroindustrias. De acuerdo con la información obtenida de la investigación, para la cosecha 2015-2016 el volumen total de leña consumida por las 67 agroindustrias evaluadas de ambos municipios fue de $37464.42 \mathrm{~m}^{3}$, con lo cual se presume que se contribuyó en un impacto aproximado sobre los bosques naturales de 378.42 ha de deforestación y una pérdida de biodiversidad incalculable, para la época en la que se realizó la investigación. Con los resultados que se presentan en este artículo se pretende establecer lineamientos estratégicos, los cuales deben ser tomados en cuenta por las autoridades e instituciones correspondientes, para promover un manejo sustentable del recurso energético (leña) y orientar el desarrollo de la agroindustria del cardamomo bajo un enfoque ecoeficiente ${ }^{1}$ y con ello promover un Desarrollo Rural Sustentable.
\end{abstract}

\section{Palabras clave}

Agroindustria, cardamomo elettaria cardamomum, ecoeficiencia, desarrollo rural sustentable, impacto ambiental.

\begin{abstract}
The study was carried out in the municipalities of Cobán and San Pedo Carchá, both in the department of Alta Verapaz, in order to analyze and characterize the agroindustrial sector that uses firewood to dry cardamom Elettaria cardamomum. The questionnaire was the instrument used to obtain valid and reliable data, necessary to reach the stated objectives. In this study, key informants were identified for the owners of agroindustries. According to the information obtained from the research, for the harvest 2015-2016 the total volume of firewood consumed by the 67 agroindustries evaluated in both municipalities was $37464.42 \mathrm{~m}^{3}$, which is presumed to have contributed to an approximate impact on the Natural forests of 378.42 ha of deforestation and a loss of biodiversity incalculable, for the time in which the research was carried out. The results presented in this article are intended to establish strategic guidelines which should be taken into account by the corresponding authorities and institutions, to promote sustainable management of energy resources (firewood) and to guide the development of the cardamom agroindustry under an Eco-efficient approach and thereby promote sustainable rural development.
\end{abstract}

1 Se refiere a la relación que existe entre un producto producido por una agroindustria y los impactos ambientales que se generan por su fabricación. Para el sector agroindustrial, este concepto implica añadir cada vez más valor a los productos, consumiendo una menor cantidad de materias primas, y a la vez generando menos contaminación a través de procedimientos sostenibles y eficientes, con el fin de promover una mejor competitividad y responsabilidad social-ambiental. 


\section{Keywords}

Agroindustry, Cardamom Elettaria cardamomum, Ecoefficiency, Integral rural development, environmental impact.

\section{Introducción}

Frente a los desafíos que conlleva el desarrollo sostenible, el estudio y análisis de las actividades económicas y productivas que han promovido la deforestación acelerada de los bosques en Guatemala, adquieren una especial relevancia. De acuerdo con el IARNA (2015), el país se encuentra en una crisis ambiental, debido a la pérdida anual de 132137 hectáreas de bosques naturales.

El cardamomo Elettaria cardamomum es una planta herbácea aromática, introducida en la región de Alta Verapaz en 1914 (CARDEGUA, 2014). Con más de 100 años de cultivarse, el cardamomo representa uno de los principales cultivos de la región. En las últimas dos décadas ha existido un incremento significativo en la producción de dicho cultivo en Guatemala, informes estadísticos sobre exportaciones reportan que desde el 2014 al presente año, se exportan más de 32,000 toneladas (BANGUAT, 2015). Esto ha originado una fuerte presión sobre los bosques naturales debido a la demanda de leña que utilizan durante la trasformación del grano de cereza a pergamino; esta situación genera un escenario desalentador para el medio rural, debido a la intervención que se tiene sobre el entorno natural y biodiversidad, por parte de la actividad agroindustrial del cardamomo en relación a la destrucción de los bosques naturales.

Debido a la falta de información en el ámbito municipal sobre aspectos relacionados con el consumo del recurso forestal (leña) utilizado por el sector agroindustrial del cardamomo, a continuación se presentan los resultados de una investigación, para ponerlos a disposición de funcionarios públicos, inversionistas privados, centros de investigación, gestores del desarrollo y la comunidad en general, con el propósito de contribuir de manera efectiva con iniciativas orientadas a mejorar la gestión de los recursos forestales en la región, a través de una eficiencia energética en el consumo de leña de las agroindustrias de cardamomo, a fin de fortalecer estrategias de desarrollo rural integral.

Este estudio aporta elementos para analizar de manera general aspectos relacionados con la utilización de leña por parte del sector agroindustrial del cardamomo Elettaria cardamomum. Se pretende que los hallazgos del mismo, puedan guiar el diseño de instrumentos de gestión ecoeficiente en este tipo de agroindustria.

\section{Marco teórico}

\subsection{Desarrollo rural sustentable}

El término "desarrollo" es un concepto con diversas interpretaciones, dependiendo del contexto en que es apreciado. El desarrollo entendido en el medio rural implica poder alcanzar condiciones de mejora social, económica, productiva y ambiental.

El desarrollo rural significa asumir el reto de desarrollar con equidad resalta la importancia de observar las dimensiones operativas del desarrollo: económicas, humanas, ecológicas, tecnológicas y culturales (Zatarian, 2008).

Orientar y cumplir con la definición de desarrollo rural sustentable en países en vías de desarrollo resulta complejo, debido a que las necesidades básicas están relacionadas a la subsistencia; la utilización excesiva de los recursos naturales y la degradación al medioambiente son consecuencia de los esfuerzos por superar la pobreza.

De acuerdo con el PNUD (2010), el país muestra indicadores totalmente desalentadores de la situación actual del sector rural. Ante tal escenario, es necesario la construcción de planteamientos con una visión integral, que consoliden un proceso de desarrollo rural y con ello contribuir con acciones que coadyuven a aminorar los impactos negativos que se presentan en el ámbito social, económico, político y ambiental, que han marcado un rezago evidente en la ruralidad guatemalteca. 
El paradigma actual en la temática del desarrollo rural sustentable, para países como Guatemala, se debe tener como objetivo principal reducir el deterioro ambiental y disminuir la pobreza. Según la Encuesta Nacional de Condiciones de Vida (ENCOVI, 2014), en Guatemala, Alta Verapaz concentra la tasa más alta de pobreza y pobreza extrema, con $82 \%$. Lo anterior pone de manifiesto que las poblaciones rurales están sumidas en un contexto de atraso, marginación y exclusión social con una marcada degradación de los recursos naturales.

Estudios recientes demuestran que los modelos de desarrollo en el país han deteriorado y, en muchos casos agotado, la base de bienes y servicios naturales. Esto provoca que las posibilidades de satisfacer necesidades de la población disminuyan en igual proporción en la que se agotan o deterioren en mayor medida el entorno natural (URL, IARNA, 2009).

\subsection{Deforestación y degradación de bosques}

En la actualidad, 30000 hectáreas de bosque se pierden en promedio cada año en el país. En solo una década, de 2004 a 2014, el país perdió una quinta parte de la cobertura forestal (Pitan, 2016).

Según el informe de balance energético del Ministerio de Energía y Minas (MEM, 2010), del total de energía consumida en el país, las fuentes energéticas que mayor consumo reportaron fue la leña con un $58.2 \%$, derivados del petróleo el $33 \%$ y electricidad el $8.8 \%$. El alto consumo de leña corresponde a que la mayor parte de la población vive en el área rural, siendo en su mayoría de escasos recursos económicos, y tanto el acceso como la disponibilidad a otras fuentes energéticas resulta difícil.

Los bosques desempeñan una función esencial en el ciclo del agua, la conservación de los suelos, fijación de carbono y la protección de los hábitats. Su gestión sostenible es crucial para lograr una agricultura sostenible y alcanzar la seguridad alimentaria (FAO, 2016). La gestión de los bosques de manera sostenible aumenta la resiliencia de los ecosistemas y de las comunidades rurales, lo que permite aprovechar de manera óptima la función de estos y a la vez beneficiarse de múltiples servicios ambientales. La deforestación en Guatemala pasó de 100 mil hectáreas anuales en el período 2001-2006 a poco más de 132 mil en el periodo 2006-2010 (IARNA-URL, 2012). Los anteriores datos confirman la alta dependencia que existe en el país sobre los recursos naturales, debido a los altos niveles de pobreza y falta de fuentes alternas de sustento, esto en los sectores rurales.

Los municipios de los departamentos de Alta Verapaz, Izabal y Petén presentan los datos más altos de pérdida de cobertura forestal en área (INAB, CONAP, URL \& UVG, 2012). En el caso de Alta Verapaz, se sabe que en las regiones productoras de cardamomo, se es partícipe en la pérdida de la cobertura forestal del departamento, debido a la demanda del energético (leña) que existe para el secado del producto.

\subsection{Agroindustria del cardamomo}

Según la Asociación de Cardamomeros de Guatemala (CARDEGUA), el cultivo es considerado como el principal activador económico en la región de Las Verapaces; a la producción de cardamomo se le atribuyen la generación de más de 50 mil empleos permanentes remunerados y 150 mil en autoempleo al año en el área rural, y aunque los precios son irregulares, los agricultores continúan dependiendo del cultivo de manera directa.

Dicho sector agroindustrial es considerado como el principal impulsor de la economía rural, tiene un enorme potencial para ser un sector mucho más dinámico. Sin embargo, hasta la fecha no se le ha dado la debida atención a la degradación del ambiente por las altas cantidades de biomasa (leña) que se utiliza como combustible para el secado del producto.

La transformación del cardamomo es el proceso donde se deshidrata el cardamomo cereza con un aproximado de $55 \%$ de humedad, hasta convertirlo a pergamino (10 - $12 \%$ humedad), conservando su coloración verde y gran proporción de su forma y tamaño. Esto se efectúa a través del uso de 
maquinaria alimentada por leña, conocidas en la región como secadoras.

Con respecto al tipo de leña, su consumo es de 16 cargas de leña, para una capacidad de 45 a 50 quintales de fruto verde (Pérez, 1981). Según Archila (2015), Director Regional de las Verapaces del Instituto Nacional de Bosques (INAB), estima que por cada cosecha de cardamomo existe un consumo aproximado de 225000 m³$^{3}$ de leña, deforestándose 2200 hectáreas, el equivalente a decir que se pierden 45 caballerías de bosque.

El canal de comercialización del cardamomo obedece a la tendencia siguiente: Productor, Intermediario, Procesador, Exportador y Consumidor; en el mercado nacional e internacional no existe algún ente regulador o fijador de los precios para el producto. Los precios a nivel nacional son determinados por los intermediarios y exportadores, por factores de oferta y demanda (Ruano, 2002). El precio es fluctuante tanto en cereza como en pergamino debido a factores de demanda y por acciones especulativas.

\subsection{Impacto ambiental de la agroindustria del cardamomo}

Existe un impacto ambiental cuando alguno de los componentes del medioambiente sufre una alteración derivada por una acción o actividad de tipo agrícola, industrial, entre otras.

La forma en la que las agroindustrias de cardamomo Elettaria cardamomum generan impactos negativos sobre el medioambiente, se relaciona al uso desmedido de leña que utilizan para la deshidratación del grano, en donde al existir alta demanda del recurso energético (leña) para el desarrollo de su actividad, se tiene una fuerte presión sobre los bosques naturales.

La cadena productiva del cardamomo repercute tanto en aspectos positivos como negativos sobre el ambiente. Según el método de cultivo, la intensidad, el manejo cultural del mismo, entre otros aspectos, pueden resultar en repercusiones negativas específicas para el medio ambiente.
Esta práctica de cómo adquieren la leña los procesadores de cardamomo Elettaria cardamomum, contribuye en gran medida a destruir los ecosistemas naturales locales. Con la deforestación que se presenta se intervienen ecosistemas naturales que en algunos casos desaparecen o se generan impactos nocivos sobre su biodiversidad.

El aprovechamiento de manera responsable y sustentable de los recursos naturales, puede llevar al sector productivo y agroindustrial a una posición privilegiada y competitiva en áreas clave para la inversión y el desarrollo. La problemática ambiental actual es muy compleja, y esa complejidad implica un cambio de actitud frente al aprovechamiento racional de los recursos, y con ello garantizar la sustentabilidad dentro de un determinado espacio natural, urbano y rural.

\subsection{Ecoeficiencia}

Se puede considerar que el concepto de ecoeficiencia surge de la relación que existe entre un producto o servicio producido por una agroindustria y los impactos ambientales que se generan por su fabricación. Para el sector agroindustrial, este concepto implica importantes beneficios ambientales y económicos para una mejor competitividad y responsabilidad social.

Una de las maneras en que se plantea el proceso de avance de los países hacia un desarrollo sostenible en la industria, o al menos, más sostenible, es adoptar un enfoque amigable con el ambiente en sus procesos (Leal, 2005).

World Business Council for Sustainable Development (WBCSD), define la ecoeficiencia como: "proporcionar bienes y servicios a un precio competitivo, que satisfaga las necesidades humanas y la calidad de vida, al tiempo que reduzca progresivamente el impacto ambiental y la intensidad de la utilización de recursos a lo largo del ciclo de vida, hasta un nivel compatible con la capacidad de carga estimada del planeta".

La anterior definición toma en cuenta que las empresas no operan de manera independiente de la 
sociedad y de los recursos naturales. Por tal razón, el desempeño del sector agroindustrial no solo debe estar inmerso en lo económico, sino también en lo social y ambiental. La ecoeficiencia se halla estrechamente ligada al desarrollo sostenible ya que equivale a optimizar tres objetivos: crecimiento económico, equidad social y valor ecológico.

Aunque no es la única causa de deforestación en la región de las Verapaces, el uso insostenible de la biomasa como combustible está causando degradación ambiental en las zonas rurales. Aplicar a nivel agroindustrial el enfoque de la ecoeficiencia para el resguardo del ambiente, implica una actitud encaminada a producir con un menor consumo de recursos naturales, reducir los residuos y atenuar la contaminación.

\section{Marco Referencial}

\subsection{Ubicación del área de estudio}

El estudio se realizó en los municipios de Cobán y San Pedro Carchá, ambos del departamento de Alta Verapaz, estos se caracterizan por tener una alta demanda de leña para el proceso de beneficiado del cardamomo Elettaria cardamomum, ya que según la base de datos de la Asociación de Cardamomeros de Guatemala (CARDEGUA), es donde se encuentra mayor área sembrada del cultivo y donde existe un mayor número de agroindustrias de cardamomo.

Figura 1

Ubicación del área de estudio

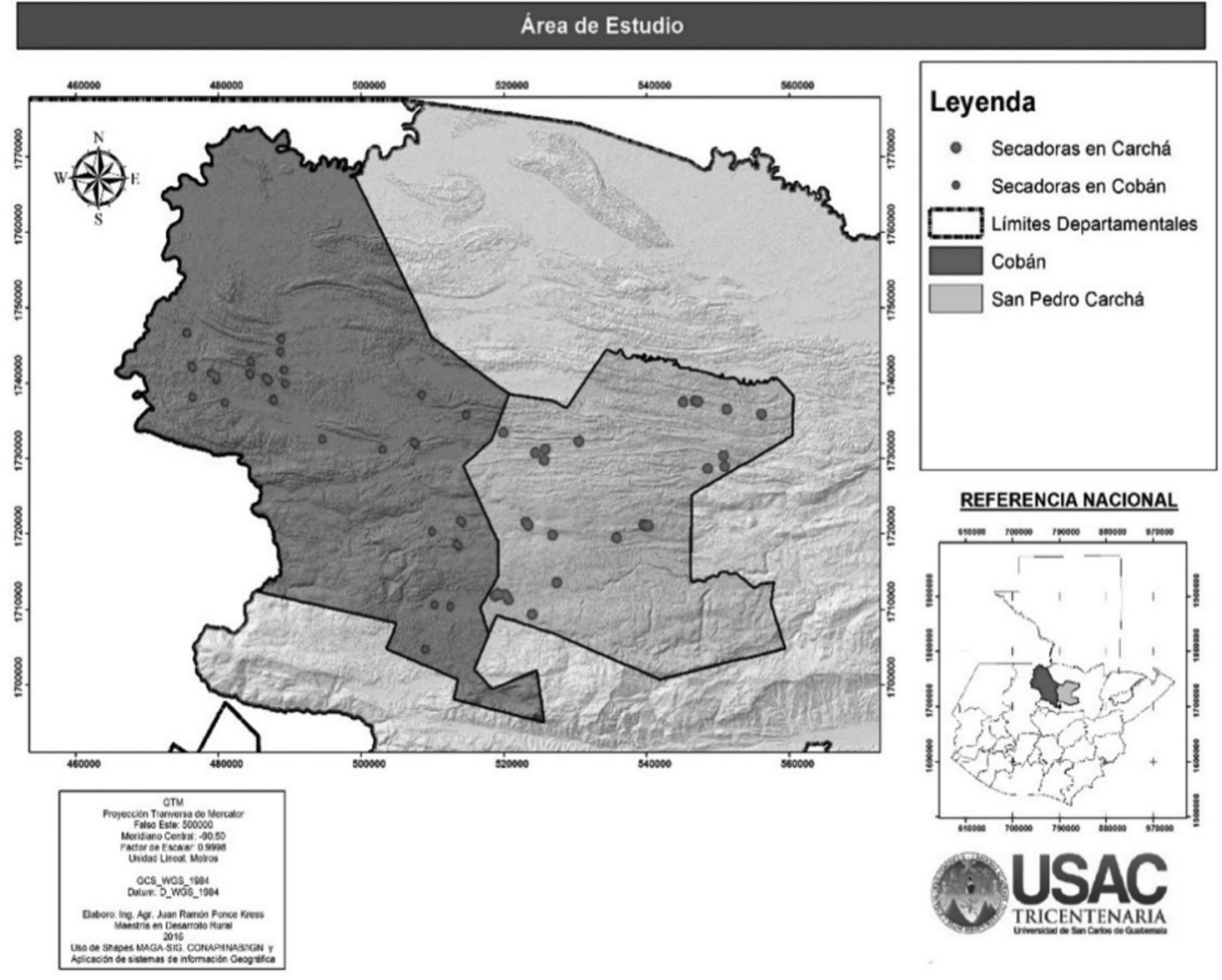

Fuente: Uso de Shapes MAGA-SIG, CONAP/INAB/IGN, aplicación de sistemas de información Geográfica y datos tomados en campo. Elaboración: Ing. Juan Ramon Ponce 


\subsection{Metodología}

Como punto de partida en esta investigación, se tomó como referencia la base de datos de CARDEGUA, en relación con el número de agroindustrias instaladas en los municipios de estudio. Según CARDEGUA (2013), en el municipio de Cobán existen 37 agroindustrias de cardamomo Elettaria cardamomum, y en el de San Pedro Carchá, hay un total de 30 agroindustrias que procesan dicho producto.

Considerando el número reportado de agroindustrias que integran la población, no fue necesario aplicar un método de muestreo. Por lo tanto se realizó un censo, que incluyó el total de beneficios reportados por CARDEGUA. La información se obtuvo mediante un cuestionario dirigido a los propietarios o administradores de las agroindustrias de cardamomo Elettaria cardamomum. La ejecución del proceso de recolección de información in situ facilitó a la investigación el alcance de objetivos planteados.

Para determinar aspectos de obra física con que cuentan las agroindustrias (beneficios) de cardamomo se utilizó una guía de observación, conformada por 16 ítems, en donde a través de una escala de medición (Likert) se estableció la condición de las mismas.

\section{Resultados y discusión}

\subsection{Caracterización del sector agroindustrial del cardamomo}

La caracterización del sector agroindustrial del cardamomo para efectos del presente estudio consideró aspectos relacionados a la capacidad instalada de cada agroindustria, en función del número de secadoras (pilas) y tipo de secadoras con que cuentan, asimismo aspectos sobre Buenas prácticas de manufacturas BPM's, las cuales se relacionan de manera indirecta en el consumo de leña. Se realizó una evaluación de la infraestructura con que cuentan para el desarrollo de su actividad agroindustrial.
En ambos municipios, se determinó que el $100 \%$ de las agroindustrias evaluadas, utilizan maquinaria para el secado de cardamomo que funciona con base de leña. No se logró identificar algún tipo de secadora que trabajara con algún otro tipo de combustible, a excepción del horno múltiple que permite el secado del producto utilizando leña y otra fuente energética alterna.

El bajo valor comercial de la leña justifica que las agroindustrias solo utilicen secadoras que funcionan a base de este combustible; sin embargo, se omite el alto costo social y ambiental que ha generado transformaciones en los espacios rurales.

Se logró identificar ecoeficiencia en la maquinaria de secado que tiene fabricado el horno con material de ladrillo en comparación con las máquinas de metal, donde existe un ahorro aproximado entre $0.39 \mathrm{~m}^{3}$ a $0.784 \mathrm{~m}^{3}$ de leña por una secada de 45 quintales de cardamomo en cereza, con este tipo de secadora, según lo manifestado.

En el municipio de San Pedo Carchá, en relación con el equipo de secado se identificó un mayor número de hornos múltiples (5), en comparación del municipio de Cobán, donde se contabilizó un horno múltiple. El uso de este tipo de maquinaria permite realizar el proceso de secado de cardamomo utilizando un recurso energético alterno a la leña, como lo es el cascabillo de café o la cáscara de macadamia.

Con el objetivo de identificar si es posible ahorrar leña en el proceso de secado de cardamomo se identificó que el 42 \% de las agroindustrias de ambos municipios, para tener un menor consumo de leña, toman en cuenta los factores siguientes: utilizar leña de buena calidad (madera dura), se logra ahorrar entre medio metro y un metro de leña por secada, no utilizar trozos de leña con pequeñas dimensiones, mezclar leña seca con verde, para disminuir consumo de metros de leña por secada e incorporar reformas a los hornos, colocándole doble ladrillo, eso mantiene mejor la temperatura, consumiendo menos cantidad de leña, entre otros aspectos. 
Se realizó una evaluación sobre conocimientos básicos de Buenas prácticas de manufacturas BPM's, las cuales se relacionan de manera indirecta en el consumo de leña. Los aspectos objeto de evaluación fueron: conocimiento técnico sobre el funcionamiento de secadora, ventilación del grano en cereza antes e iniciar el proceso de secado, nivelación constante del producto en pila y aspectos de capacitación del personal involucrado en el proceso de beneficiado.

Para el desarrollo de las actividades que realizan las agroindustrias de cardamomo contar con la infraestructura adecuada, maquinaria y equipo suficiente son aspectos relevantes para lograr una mejora operativa, en referencia a este tema existen grandes carencias en este ámbito. En términos generales la mayoría de beneficios de cardamomo se caracteriza por tener instalaciones e infraestructura rústica para el desarrollo de su actividad comercial.

\subsection{Estimación del volumen de leña consumida y su relación con la pérdida de cobertura forestal}

\subsubsection{Volumen de leña utilizada en el proceso de secado del cardamomo}

Parte fundamental para estimar el volumen de leña utilizada por las agroindustrias, consideradas como objeto de estudio, fue conocer el consumo de leña por una secada de 45 q de cereza, número de quintales de cereza procesados y número de quintales pergamino obtenidos como resultado del proceso de una secada (rendimiento).

De acuerdo con la información proporcionada por los 67 propietarios de las agroindustrias, se logró identificar un rendimiento de inicio de cosecha, media cosecha y final de la misma, obteniendo un rendimiento promedio para la cosecha en la que se realizó el estudio. El resumen de esta información se presenta en la tabla siguiente:

Tabla 1

Rendimientos del proceso cereza-pergamino del cardamomo, en agroindustrias evaluadas en los municipios de Cobán y San Pedro Carchá, Alta Verapaz

\begin{tabular}{lcccc} 
& Límite inferior & Promedio & Límite superior & Municipio \\
\hline $\begin{array}{l}\text { Relación cardamomo cereza/ } \\
\text { cardamomo pergamino }(\mathbf{q} / \mathbf{q})\end{array}$ & 4.81 & 5.10 & 5.39 & Cobán \\
\hline
\end{tabular}

Fuente: Elaboración propia.

Para ambos casos se procedió a calcular la desviación estándar, para conocer un rango de valores del rendimiento. Para Cobán se tiene un rango entre 4.81 y 5.39 quintales de cereza para obtener 1 quintal de pergamino. En San Pedro Carchá, el rango está entre 4.95 y 5.41 q de cereza por 1 q de pergamino.

La variación de los rendimientos, se relaciona a que los frutos del cardamomo Elettaria cardamo- mum no llegan a su estado de madurez al mismo tiempo, la cosecha se realiza en 3 o 4 cortes de acuerdo con su maduración. Puede estimarse que mientras más maduro (sazón) está el producto, el rendimiento tiende a disminuir; y mientras más inmaduro (tierno) está el grano, el rendimiento que se obtiene en el proceso de secado tiende a subir. Mientras más bajo sea el rendimiento que se obtiene después del proceso de secado, se consigue un cardamomo con mayor densidad. 
De acuerdo con la información obtenida por parte de los propietarios de las agroindustrias de cada uno de los municipios en estudio, se logró determinar la producción anual para el período evaluado, el rendimiento del proceso de secado y el consumo de leña para la producción de un quintal pergamino. Estos datos se presentan en la tabla siguiente.

Tabla 2

Resumen del consumo total de leña consumida por las sesenta y siete agroindustrias de cardamomo para la cosecha 2015-2016 investigadas

\begin{tabular}{ccccccc} 
Municipio & $\begin{array}{c}\text { Número } \\
\text { beneficios }\end{array}$ & $\begin{array}{c}\text { Quintales } \\
\text { de cereza } \\
\text { procesados } \\
\text { cosecha }\end{array}$ & $\begin{array}{c}\text { Rendimiento } \\
\text { promedio }\end{array}$ & $\begin{array}{c}\text { Quintales } \\
\text { pergamino } \\
\text { cosecha } \\
2015-16\end{array}$ & $\begin{array}{c}\mathrm{m}^{3} \text { de leña } \\
\text { para obtener } \\
1 \text { q de } \\
\text { pergamino }\end{array}$ & $\begin{array}{c}\text { Consumo total } \\
\text { de leña }\left(\mathrm{m}^{3}\right)\end{array}$ \\
\hline Cobán & 37 & 323405 & 5.10 & 63412.74 & 0.33 & 20926.68 \\
\hline Carchá & 30 & 194700 & 5.18 & 37586.87 & 0.44 & 16538.22 \\
\hline Total & 67 & & & & & 37464.90 \\
\hline
\end{tabular}

Fuente: Elaboración propia.

En la tabla anterior se aprecia el volumen de leña consumida en el período 2015-2016, por el total de las 67 agroindustrias de cardamomo que se identificaron en ambos municipios, donde se tiene un consumo total de $37464.90 \mathrm{~m}^{3}$ de leña. Con este dato es posible realizar una estimación de la posible superficie deforestada de ambos municipios por el consumo de leña para el secado del cardamomo Elettaria cardamomum.

\subsubsection{Estimación de la pérdida de cobertura forestal}

Para calcular el área posiblemente deforestada se parte del supuesto siguiente: la estimación de volumen aprovechable para leña en bosques es de 95 $\mathrm{m}^{3} /$ ha y fuera de estos es de $13 \mathrm{~m}^{3} / \mathrm{ha}$. Los bosques de latifoliadas tienen un potencial de aporte de 99 $\mathrm{m}^{3} / \mathrm{ha}$; mientras que los bosques de coníferas $61 \mathrm{~m}^{3} /$ ha, y los bosques mixtos $59 \mathrm{~m}^{3} /$ ha (CONAP, 2009).

Para este estudio se considera el dato de $99 \mathrm{~m}^{3 /}$ ha, ya que la leña que se utiliza para el proceso del cardamomo proviene en su mayoría de bosques de latifoliadas. El consumo total de leña de las 37 agroindustrias evaluadas en el municipio de Cobán, para el período en que se realizó el estudio fue de $20926.68 \mathrm{~m}^{3}$. El municipio de San Pedro Carchá, refleja un consumo de $16538.22 \mathrm{~m}^{3}$ de leña por las 30 agroindustrias de cardamomo Elettaria cardamomum.

Con base en lo anterior, las 67 agroindustrias tuvieron un consumo aproximado de $37464.90 \mathrm{~m}^{3}$ de leña para la cosecha correspondiente al período 2015-2016. Por consiguiente, al tomar como referencia un promedio de volumen aprovechable para bosques de latifoliadas de $99 \mathrm{~m}^{3} / \mathrm{ha}$, se estima que son 378.42 ha por año de deforestación en los municipios de Cobán y San Pedro Carchá, por concepto de leña, para el secado de cardamomo Elettaria cardamomum.

En las figuras 2 y 3 se presenta de manera gráfica un comparativo de la dinámica forestal de diferentes periodos en cada uno de los municipios donde se realizó el estudio. En el mismo se puede apreciar cómo la cobertura forestal ha ido en disminución, teniendo en la actualidad menor cantidad de hectáreas de bosque. Es importante indicar que el consumo de leña para la agroindustria de cardamomo no es el único responsable por la deforestación que persiste hoy en día, existen otros factores y actividades, las cuales demandan de leña como fuente de energía para el desarrollo de sus actividades. 
Figura 2

Mapa de Dinámica Forestal del municipio de San Pedro Carchá

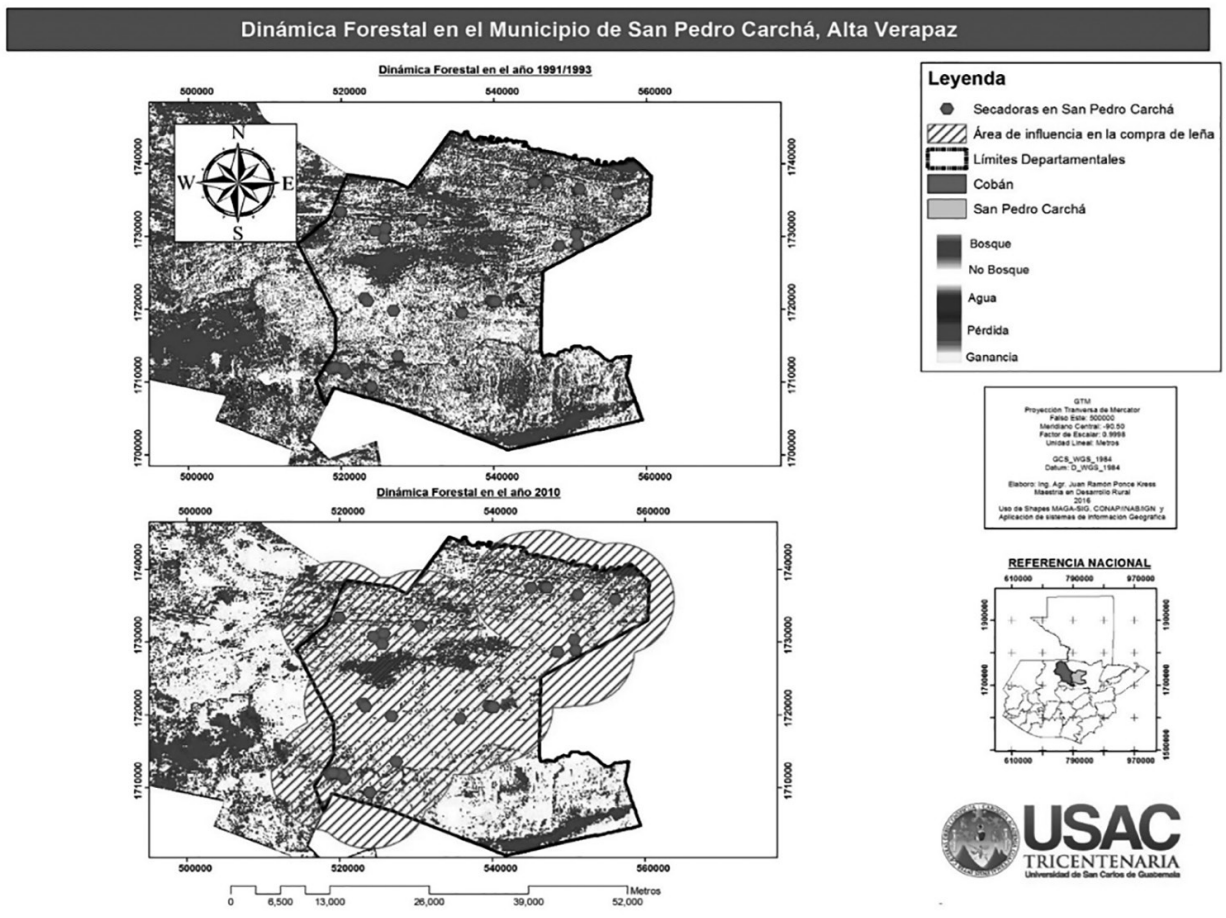

Fuente: Elaboración propia.

Figura 3

Mapa de Dinámica Forestal del municipio de Cobán

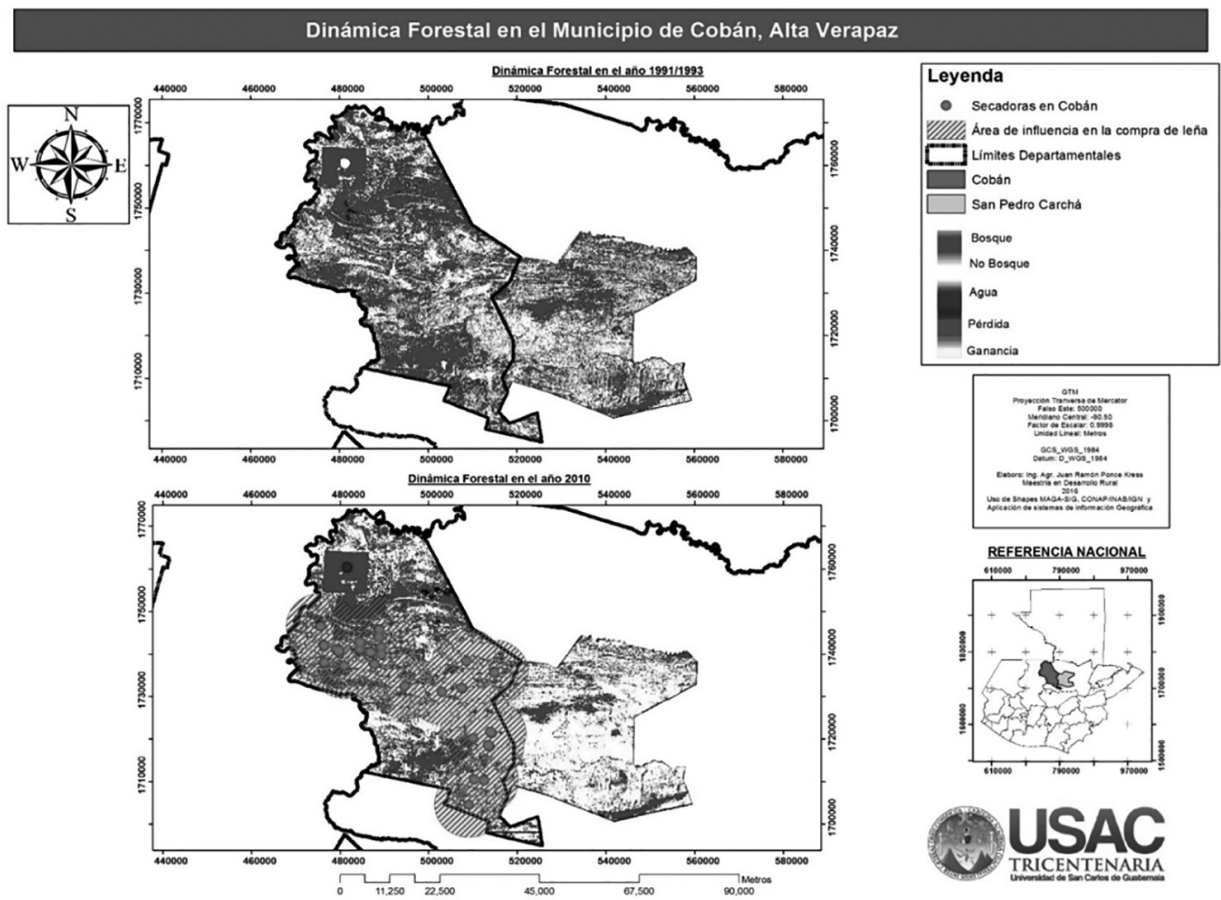

Fuente: Elaboración propia. 
4.2.3 Patrones relacionados con el consumo, precio, forma de abastecimiento, especies de uso y otros factores vinculados a la leña utilizada por las agroindustrias de cardamomo Elettaria cardamomum

\section{a. Descripción de características de consumo}

En relación con el consumo de leña, este se describe según el tipo de energético, de producto, forma de abastecimiento y periodicidad de compra. Estas características se presentan en la figura 4.

Figura 4

Características del consumo y abastecimiento de leña, agroindustrias de cardamomo, en Cobán y San Pedro Carchá, Alta Verapaz
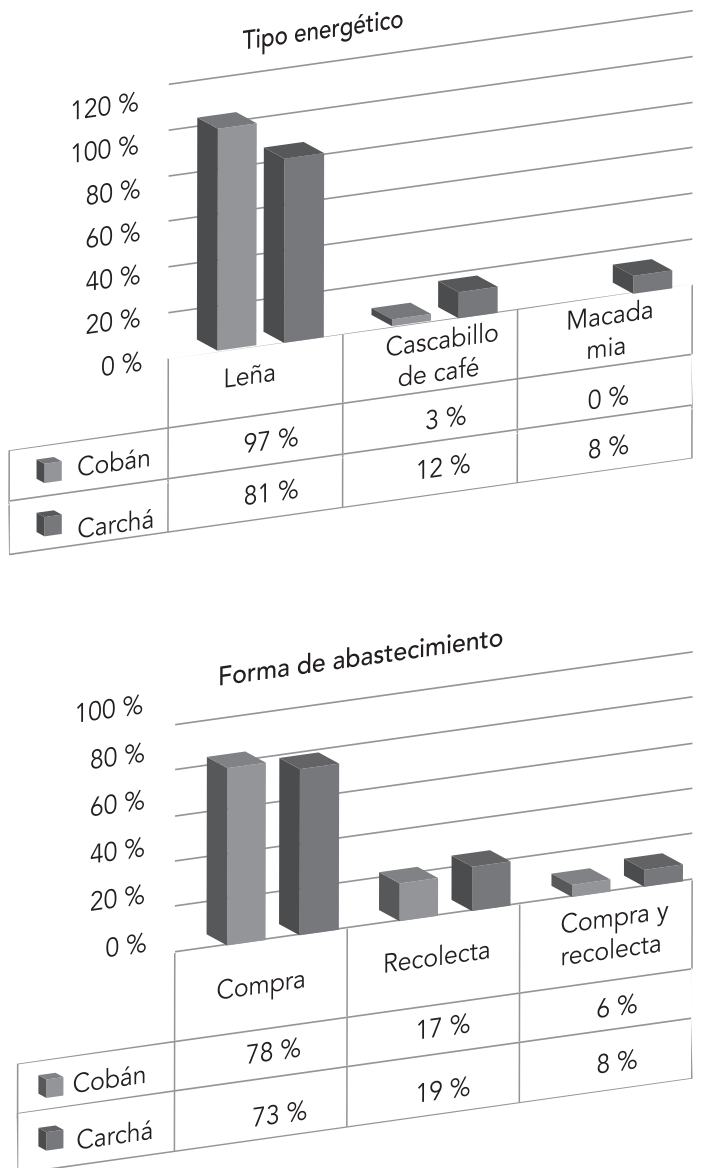
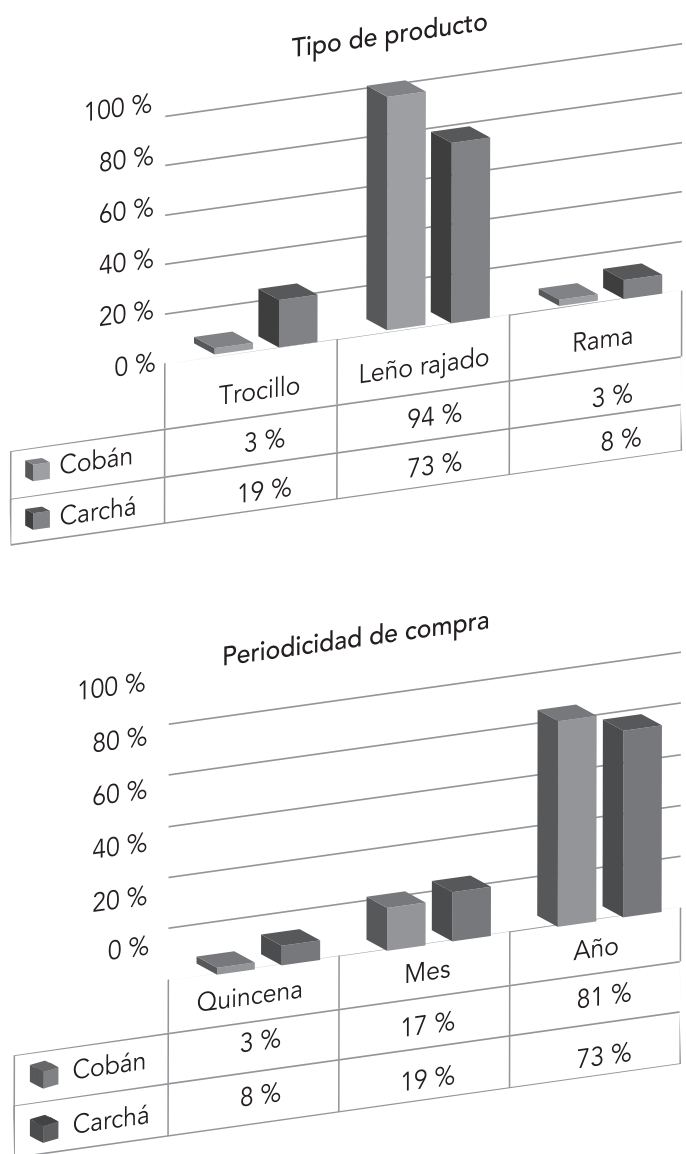

Fuente: Elaboración propia.

El abastecimiento por compra es el que más predomina en las agroindustrias de cardamomo Elettaria cardamomum. La recolección se da por parte de los propietarios de las agroindustrias que tienen parcelas con montaña. En un mínimo porcentaje (6-8 \%) existen casos donde la compra se combina con la recolección. 
Las agroindustrias se abastecen de leña en época de verano, entre los meses de marzo y abril, debido a que en esos meses es cuando pueden acceder a la montaña y el acarreo de leña es factible. Más del 70 compra la leña para toda la cosecha, entre el 17 y $19 \%$ la compra por mes y un mínimo porcentaje (3-8 \%) cada 15 días. Para los beneficios que adquieren la leña para una cosecha se llevan en promedio 2 meses para el abastecimiento de la misma.

La distancia que se recorre para el abastecimiento de leña está relacionada con una mayor o menor disponibilidad del recurso forestal. En este aspecto la distancia entre las fuentes que abastecen de leña y las agroindustrias de cardamomo Elettaria carda- momum, se encontró una alta variabilidad. Se indicaron distancias mínimas de 2 kilómetros hasta un máximo de $40 \mathrm{~km}$; en promedio la distancia que tienen que recorrer para abastecerse de leña es entre $7.7 \mathrm{~km}$. En un mínimo porcentaje hay agroindustrias que compran la leña puesta en el beneficio, esto se observa en las agroindustrias ubicadas en el sector urbano de los municipios en estudio (12\%).

\section{b. Procedencia de la leña}

Para determinar la procedencia de la leña se consideraron las variables de origen por tipo de bosque y tipo de predio. Los resultados se presentan en las figuras 5 y 6 .

Figura 5

Procedencia de leña que utilizan las agroindustrias de cardamomo según tipo de predio
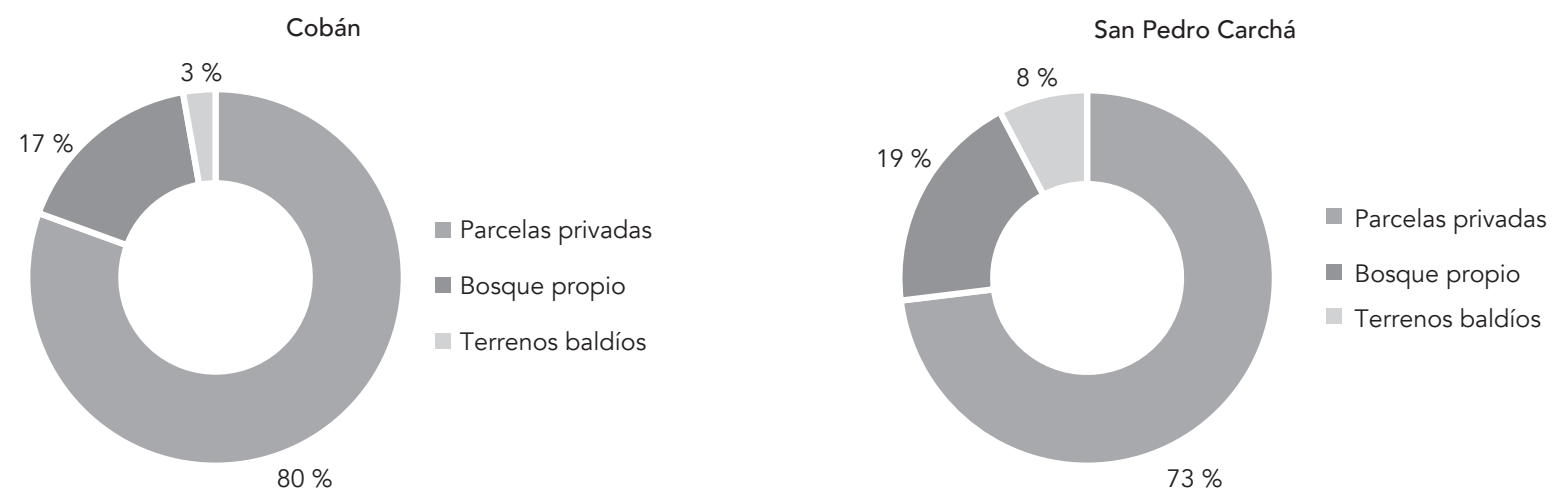

Fuente: Elaboración Propia.

Figura 6

Procedencia de leña utilizada por agroindustrias de cardamomo Elettaria cardamomum, según tipo de bosque

San Pedro Carchá

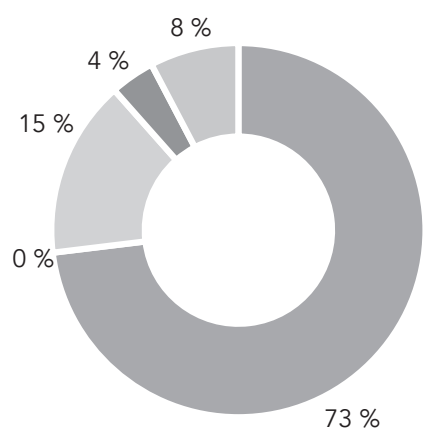

Bosque natural

Sistema agroforestal

No sabe

Plantación

Árboles dispersos

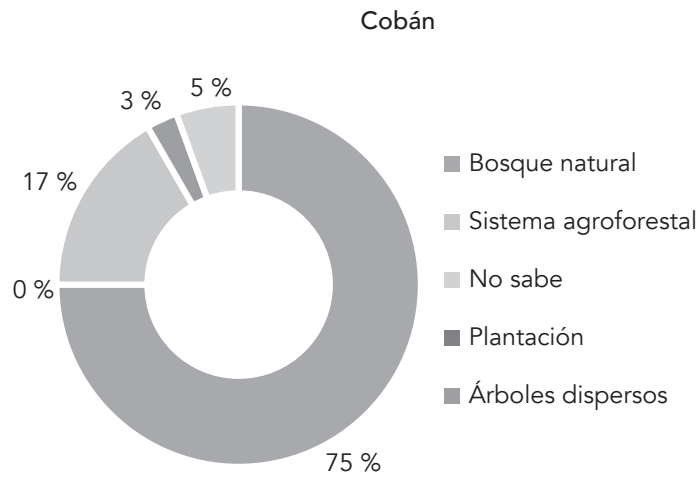

Fuente: Elaboración propia. 
De acuerdo con los resultados obtenidos, la principal fuente de donde se abastece de leña la agroindustria de cardamomo es el bosque natural (70\%). Un $17 \%$ de agroindustrias ubicadas en Cobán, manifestó que la biomasa la obtiene de sistemas agroforestales, un $3 \%$ de árboles dispersos, $5 \%$ desconoce su procedencia. Se resalta que ningún beneficio obtiene leña de una plantación forestal artificial, esto puede ser atribuido a que no existen plantaciones forestales con fines energéticos en la región.

Para el municipio de San Pedro Carchá los resultados son similares, un $73 \%$ adquiere la leña de un bosque natural, un $15 \%$ indicó que una de las formas de cómo obtienen la leña es a través de un sistema agroforestal, este es un sistema productivo que integra el manejo de un cultivo con árboles maderables, un $4 \%$ manifestó que proviene de árboles dispersos, el $8 \%$ desconoce de dónde proviene la leña, y como en el caso del otro municipio ninguna agroindustria adquiere leña proveniente de una plantación forestal artificial.

Es notorio que el aprovechamiento de leña utilizada con fines energéticos ha agravado aún más la pérdida de los bosques naturales. La principal fuente de abastecimiento de las industrias es el bosque natural, las plantaciones forestales aprovechadas constituyen solamente el $6 \%$ del volumen demandado por las industrias (Cabrera, 2003). En países de América del Sur, por ejemplo, para finales de los años noventa, la industria se abastecía en un $95 \%$ de las plantaciones forestales artificiales.

El establecimiento de plantaciones forestales energéticas, debería en un mediano y largo plazo atender la demanda del recurso energético de la agroindustria, y así reducir la presión sobre los bosques naturales. Una plantación de este tipo, se refiere a la siembra de una masa arbórea, cuyo aprovechamiento cíclico y programado asegure un abastecimiento permanente de leña.

Tanto los bosques como los sistemas agroforestales, cumplen un rol fundamental en las estrategias de vida de las familias rurales, debido a que constituyen una fuente de abastecimiento de leña, ma- dera, agua, áreas de pastoreo y otros recursos que de ahí se derivan (Silvel et al. 2009).

La extracción de leña se encuentra relacionada de manera directa a la pobreza de las poblaciones rurales, que necesitan explotar los ecosistemas naturales para obtener recursos energéticos. Asimismo se ven en la necesidad de abrir nuevos espacios para la agricultura y crianza de ganado, aspectos que coinciden con la tipología agraria de Guatemala, donde el sector productivo se encuentra en una condición de infrasubsistencia y subsistencia.

A pesar de que existen ciertas regulaciones para el manejo de los recursos naturales en el país, hay vacíos legales para en la comercialización de la biomasa forestal.

El alto grado de informalidad con que se comercializa la leña, se refleja en los resultados obtenidos, donde el $83 \%$ de agroindustrias compra la leña sin algún tipo de documento legal, y un (19 \%) la adquiere con licencia. Esto para el caso de las agroindustrias ubicadas en el municipio de Cobán.

Según Silvel et al. (2009), las trabas, los costos y las demoras de las gestiones forestales, estimulan la ilegalidad, ya que en la mayoría de municipios del país, se ha reducido el número de planes de manejo, pero ha crecido la extracción y transporte ilegal de productos forestales. La ausencia de planes de manejo es la principal amenaza de la sobrevivencia y conservación de los bosques.

\section{c. Especies de leña de mayor preferencia}

De acuerdo con lo indicado por los propietarios o administradores, se logró identificar las especies de leña de mayor preferencia para el proceso de secado del cardamomo Elettaria cardamomum, mencionando el nombre común de la especie, para luego investigar el nombre científico de cada una de las especies mencionadas. Se logró determinar que el $95 \%$ de estas especies provienen de bosques latifoliados, con procedencia de bosque secundario. 
En la siguiente tabla se enlistan las especies de mayor uso y preferencia en el municipio de San Pedo Carchá, definiendo el poder calorífico de cada una de ellas. El poder calorífico de la leña varía en función de la clase de leña utilizada, la parte del árbol de la cual proviene (rama o tronco) y contenido de humedad de la misma. En la agroindustria de car- damomo, existe mayor preferencia en la utilización de leña que se caracteriza por tener un alto poder calorífico. Las especies más utilizadas se caracterizan por su alta densidad, capacidad para quemarse de manera lenta y una adecuada producción de brasas.

Tabla 3

Listado de las tres especies de leña más comercializadas y utilizadas por las agroindustrias ubicadas en el municipio de San Pedro Carchá

\section{$\begin{array}{lll}\text { Nombre común } & \text { Nombre científico } & \text { Poder calorífico } \mathrm{kcal} / \mathrm{kg}\end{array}$}

1. Liquidámbar

2. Encino

3. Canchán
Liquidambar styraciflua

Quercus spp

Terminalia amazonia
$5977 \mathrm{kcal} / \mathrm{kg}$

$4658 \mathrm{kcal} / \mathrm{kg}$

$4572 \mathrm{kcal} / \mathrm{kg}$

Fuente: Elaboración propia.

En la tabla 4, se enlistan las especies de mayor preferencia en la región del municipio de Cobán. La principal especie de leña que buscan y consumen es el tamarindo Dialium guianense (Aubl.) Sand- with., seguido del canchán Terminalia amazonia, y liquidámbar Liquidambar styraciflu). Mientras más densa es la leña, mayor poder calorífico.

Tabla 4

Listado de las tres especies de leña más comercializadas y utilizadas por las agroindustrias ubicadas en el municipio de Cobán

\begin{tabular}{llc}
\multicolumn{1}{c}{ Nombre común } & \multicolumn{1}{c}{ Nombre científico } & Poder calorífico kcal/kg \\
1. Tamarindo & Dialium guianense (Aubl.) Sandwith. & $4850 \mathrm{kcal} / \mathrm{kg}$ \\
2. Canchán & Terminalia amazonia & $4572 \mathrm{kcal} / \mathrm{kg}$ \\
3. Liquidámbar & Liquidambar styraciflua & $5977 \mathrm{kcal} / \mathrm{kg}$ \\
\hline
\end{tabular}

Fuente: Elaboración propia.

\section{d. Posible escasez de leña en un futuro}

En términos generales, se determinó que en el sector agroindustrial de cardamomo hay preocupación por el abastecimiento futuro de leña. Hay un reconocimiento generalizado de la visible reducción del recurso forestal. Los entrevistados indicaron que el abastecimiento de leña se ha vuelto más difícil en lo últimos años. Como un efecto de la escasez, se expresó que la leña cada año estaba más cara. Este problema afecta principalmente a las agroindustrias de cardamomo que adquieren la leña mediante compra. Se pudo establecer que más del $70 \%$ de agroindustrias investigadas obtienen la leña de esta forma.

Con el objetivo de establecer si han existido acciones por parte de los propietarios de las agroindus- 
trias en la siembra de árboles, como una responsabilidad ambiental por la actividad agroindustrial que realizan, en ambos municipios más del $90 \%$ indicó que nunca han plantado árboles para producir su propia leña. Esto denota la nula conciencia ambiental de dicho sector empresarial.

El $100 \%$ de los propietarios o encargados de las agroindustrias de cardamomo, manifestaron que el uso de leña para el secado del cardamomo genera impactos negativos al ambiente. El principal y quizás el más irreversible es la contribución de esta agroindustria en la tala indiscriminada de los bosques naturales, que se traduce en una significativa destrucción del recurso forestal y biodiversidad.

Sin embargo, la leña es uno de los combustibles preferidos en el sector agroindustrial del cardamomo en la región, debido a su menor costo, en comparación con otros combustibles. Otra de las ventajas que caracteriza a la leña es que es un producto local, esto significa que en un alto porcentaje se produce y comercializa en las mismas zonas donde se consume.

Frente al problema de la escasez de leña para un futuro, se les cuestionó a los propietarios o encargados de las agroindustrias si ellos tenían ideas acerca de cómo ayudar a solucionar el problema de escasez de leña que comienza a manifestarse en el área de acción donde realizan su actividad industrial. Las respuestas fueron las siguientes: plantar árboles para leña (38\%), cambiar fuente energética (17\%), mejorar eficiencia de maquinaria (14\%) y del $31 \%$ restante no se obtuvo respuesta.

En el cuestionario se le preguntó a los propietarios de las agroindustrias si estarían dispuestos a plantar árboles para la leña como una posible solución; El 71 \% (44 propietarios) contestó de manera afirmativa.

Entre los factores que pueden llegar a ser una limitante para los propietarios de las agroindustrias de cardamomo, en la siembra de árboles para leña, las personas entrevistadas mencionaron lo siguiente: falta de asistencia técnica (30 \%), largo tiempo de aprovechamiento (25\%), no saben qué especie de árboles sembrar (18\%) y falta de terreno (7 \%). Del otro $20 \%$ no se obtuvo ninguna respuesta.

\subsubsection{Diseño de una propuesta ecoeficiente para el uso del recurso energético (leña), en la agroindustria de cardamomo Elettaria cardamomum}

El diseño de la propuesta ecoeficiente para el sector agroindustrial del cardamomo, tiene como principal fin el desarrollo de iniciativas regionales, las que están relacionadas específicamente con el objetivo 9 (Industria, innovación e infraestructura), objetivo 11 (Ciudades y comunidades sostenibles), objetivo 12 (Producción y consumo responsable), objetivo 13 (Acción por el clima) y el objetivo número 15 (Vida de ecosistemas terrestres); estos son parte de los 17 objetivos que conforman los ODS, planteados en la Agenda de Desarrollo Global post 2015.

Los diecisiete Objetivos de Desarrollo Sostenible son nombrados de carácter integrado e indivisible, los progresos hacia la agricultura sostenible, la seguridad alimentaria y la gestión forestal sostenible, elementos básicos de los ODS, deben alcanzarse en paralelo (FAO, 2016).

Un plan de gestión es un instrumento orientador y directriz que establece un conjunto de actividades ordenadas y planificadas, para resolver la problemática y aprovechar las potencialidades de un territorio en forma sostenible y que provea bienestar a las poblaciones (Jiménez, 2014).

Con base en los hallazgos de la investigación, se realizó un análisis de los mismos, con el propósito de realizar una propuesta de un plan de gestión ecoeficiente. Esta contempla líneas de acción y actividades clave que pretenden facilitar o solucionar la problemática identificada en el sector agroindustrial cardamomero respecto al uso de leña. La propuesta contempla los siguientes 5 ejes estratégicos.

Eje 1. Fortalecimiento institucional de las agroindustrias de cardamomo: tiene como objetivo principal promover la agrupación del sector Mipyme 
articulado en el beneficiado del cardamomo Elettaria cardamomum. Contempla las líneas de acción siguientes: Organizar a los actores del sector agroindustrial de cardamomo y gestionar fuentes de apoyo para el referido sector. A través de la organización que se pretende lograr, este sector agroindustrial puede ser artífice de su propio desarrollo, en los diferentes ámbitos relacionados a su actividad comercial.

Eje 2. Promoción del manejo sustentable de los recursos naturales: orientado a buscar alternativas de utilización de los recursos sin degradar el ecosistema. Este eje considera las líneas de acción siguientes: restauración de ecosistemas, incorporar y aplicar criterios ambientales que contribuyan a controlar la deforestación e impulsar procesos de formación y participación ciudadana. Este eje persigue detener y revertir los procesos de deterioro en las regiones cercanas a las agroindustrias de cardamomo, mediante la restauración y recuperación de ecosistemas que han sido transformados.

Eje 3. Fomento a la inversión de producción de leña: contribuir con el manejo de los bosques impulsando la reforestación y promoviendo la implementación de bosques energéticos y sistemas agroforestales. Las líneas de acción están enfocadas a fomentar la inversión en plantaciones energéticas, promover sistemas agroforestales y fortalecer el mercado de leña destinado para el sector agroindustrial del cardamomo. Con este eje se pretende disponer de plantaciones cuyos fines estén dirigidos a satisfacer la demanda existente de leña.

Eje 4. Promoción del uso de sistemas de secado eficientes de consumo de leña: incentivar a las agroindustrias de cardamomo Elettaria cardamomum a la adopción de tecnologías de combustión de leña más eficiente y más amigables con el medioambiente. Considera las líneas de acción siguientes: identificar tecnologías ecoeficientes en secadoras de cardamomo y fomentar la construcción y uso de las mismas. Con la implementación de las actividades de este eje se pretende lograr una eficiencia energética en los sistemas de secado que utilizan en la actualidad las agroindustrias de cardamomo.
Eje 5. Uso de fuentes energéticas alternas de energía para el secado de cardamomo: identificar y promover la utilización de fuentes energéticas alternas para el secamiento del grano en cereza. Las líneas de acción de este eje se centran en un proceso de investigación y desarrollo, y en gestionar una certificación de las agroindustrias. El objetivo principal es que el sector agroindustrial que utiliza leña para el secado de cardamomo, haga uso de un tipo de energía que no consuma de manera excesiva recursos y perturbe los componentes bióticos y abióticos de los ecosistemas.

\section{Conclusiones}

En ambos municipios donde se realizó el estudio, existe una alta dependencia sobre el recurso energético (leña), debido a que es el principal combustible utilizado por las secadoras de cardamomo Elettaria cardamomum. En un mínimo porcentaje (9\%) se identificaron agroindustrias que utilizan fuentes alternas de energía (cascabillo de café y cáscara de macadamia) para realizar el proceso de secado.

Según el equipo de secado que utilizan las agroindustrias de cardamomo Elettaria cardamomum es posible atribuir una variación en el consumo de leña de 0.30 - $0.784 \mathrm{~m}^{3}$ para una secada de 45 quintales de cereza, debido a factores de diseño de las secadoras y material de fabricación, lo cual permite tener un menor gasto de biomasa.

En los sitios de estudio, el sector agroindustrial de cardamomo presenta una serie de deficiencias en su infraestructura para el desarrollo de su actividad agroindustrial; sin embargo, existen aspectos que pueden ser corregidos para mejorar la competitividad de dicho sector; asimismo, las deficiencias observadas en relación con las buenas prácticas de manufactura en el beneficiado, demuestran un bajo nivel tecnológico en el proceso de secado.

Se estima que el volumen total de leña consumida en el período 2015-2016, por el total de las 67 agroindustrias (beneficios) de cardamomo Elettaria cardamomum, que se evaluaron fue de 37464.90 $\mathrm{m}^{3}$ de leña, $20926.68 \mathrm{~m}^{3}$ corresponden al munici- 
pio de Cobán, y $16538.22 \mathrm{~m}^{3}$ al municipio de San Pedro Carchá.

Tomando como referencia un promedio de volumen aprovechable para bosques de latifoliadas de $99 \mathrm{~m}^{3} / \mathrm{ha}$, y el consumo total de leña $37464.90 \mathrm{~m}^{3}$ para lo cosecha 2015-2016 de cardamomo Elettaria cardamomum; en los municipios de estudio, se estima que se generó impacto en la contribución a la deforestación sobre los bosques naturales equivalente a 378.42 ha deforestadas.

Para este estudio, se determinó que la cantidad de leña necesaria para obtener un quintal $(50 \mathrm{~kg})$ de cardamomo en pergamino, es de $0.33 \mathrm{~m}^{3}$ para el municipio de Cobán, y de $0.44 \mathrm{~m}^{3}$ para el caso de San Pedro Carchá. En relación con los rendimientos que se obtienen después del proceso de beneficiado del cardamomo, se determinó que en Cobán se tiene en promedio un rendimiento de 5.10 y 5.18 para las agroindustrias de San Pedro Carchá.

\section{Referencias bibliográficas}

Banco de Guatemala. (2015). Estadísticas de los 25 principales productos de exportación. Disponible en http://www.banguat.gob.gt.

Cabrera, C. (2003). Plantaciones forestales: oportunidades para el desarrollo sostenible. Universidad Rafael Landívar. Guatemala: Instituto de Agricultura, Recursos Naturales y Ambiente.

FAO (Organización de las Naciones Unidas para la Alimentación y la Agricultura) (2016). El estado de los bosques del mundo 2016. Roma: FAO.

Guía para la ecoeficiencia. Disponible en http// www.forumambiental.org/pdf/guiacast.pdf. (Noviembre 22 del 2015).

INAB (Instituto Nacional de Bosques) (2012). Mapa de cobertura forestal de Guatemala 2010 y dinámica de la cobertura forestal 2006-2010. Guatemala: INAB.
INE (Instituto Nacional de Estadística). (2014). Encuesta Nacional de Condiciones de vida. Guatemala: INE.

Jiménez, F. (2014). Planificación del manejo y gestión de cuencas hidrográficas. Costa Rica: Centro Agronómico Tropical de Investigación y Enseñanza.

Leal, J. (2005). Ecoeficiencia: marco de análisis, indicadores y experiencias. Santiago de Chile: División de Desarrollo Sostenible y Asentamientos Humanos.

MEM (Ministerio de Energía y Minas) (2010). Informe balance energético año 2010. Guatemala: MEM.

Pérez, A. (1981). Costos de beneficiado del cardamomo según los tipos de secadoras comúnmente utilizados en el departamento de Alta Verapaz. Tesis. Ingeniero Agrónomo. Universidad de San Carlos de Guatemala. Guatemala: Facultad de Agronomía.

PNUD (Programa de las Naciones Unidas para el Desarrollo). (2010). Guatemala: hacia un estado para el desarrollo humano, Informe Nacional de Desarrollo Humano. Guatemala. Editorial Sur.

Pitan, E. (2016). Cifras de Guatemala. Prensa Libre, pp. 6-7.

Ruano, R. (2002). El cultivo del cardamomo (Elettaria cardamomum), en Guatemala. Guatemala: Instituto de Ciencia y Tecnología Agrícolas.

Silvel, E., Larson, A. y J. Mendoza. (2009). Tenencia de la tierra, Bosques y medios de vida en el altiplano occidental de Guatemala. Guatemala: Editorial de Ciencias Sociales.

Tecnología Eco-Eficiente. Disponible en https://alianzaporelclima.wordpress. com/2016/10/24/tecnologias-eco-eficiente. (Junio 10 del 2016). 
URL (Universidad Rafael Landívar). (2009). Evaluación de la sostenibilidad del desarrollo de Guatemala. Período 1990-2008. Guatemala: Instituto de Agricultura, Recursos Naturales y Ambiente.

URL (Universidad Rafael Landívar). (2012). Perfil Ambiental de Guatemala, Vulnerabilidad local y creciente construcción de riesgo. Guatema- la: Instituto de Agricultura, Recursos Naturales y Ambiente.

Zatarian, D. (2008). Desarrollo rural y conservación de recursos naturales: El ejido Sierra de Juárez, Baja California. Tesis Maestría en Administración Integral del Ambiente. Instituto de investigación científica y docencia COLEF. México: CICESE. 
